\title{
森林溪流倒木生态学研究进展
}

\author{
魏晓华 1 代力民 2 \\ (1 不列颠哥伦比亚大学地球与环境科学系, 基隆拉, V1V1V7, 加拿大) \\ (2 中国科学院沈阳应用生态所, 沈阳 110016)
}

摘 要 溪流倒木是指在河流中长度大于 $1 \mathrm{~m}$ 、直径大于 $10 \mathrm{~cm}$ 的死木。溪流倒木在森林河流中 (特别是较小的河 流中)是一个常见且重要的结构成分。该文综述了近 30 年溪流倒木的研究成果 (主要来自北美) ,总结溪流倒木在 河流形态、碳循环、泥沙与养分拦截、水生生境的形成、水生生物多样性等方面的生态功能 , 倒木的时间动态性与空 间变异性，以及干扰(包括自然干扰与人为干扰)与倒木存留量及分布的关系。此外, 该文也探讨了溪流倒木的生 态管理模式及未来研究方向。大量的研究证明, 溪流倒木对森林水生生态系统具有重要的生态功能, 但它的存留 量、分布以及它的生态意义因所研究的森林生态系统、河流大小不同而异。随着河流宽度的增加, 倒木的存留量及 它对河流的影响减少, 并且倒木的分布以单个为主变为聚集体为主。单个倒木的直径则随河流宽度增加而增大。 倒木也呈现十分明显的时间动态性, 而这种动态往往是由大规模毁灭性的森林干扰 (火、风倒等) 所驱动的。研究 倒木的时空变异性及自然干扰与人为干扰对倒木的不同影响对于保持倒木的生态功能是十分必要的。该文还对 中国开展溪流倒木的生态研究提出一些建议。

关键词 溪流倒木 生态功能 倒木时空变异性 千扰

\section{REVIEW OF ECOLOGICAL RESEARCH ON IN-STREAM WOOD}

\author{
WEI Xiao-Hua ${ }^{1}$ and DAI Li-Min ${ }^{2}$ \\ (1 Earth and Environmental Science Department, University of British Columbia , Okanagan, British Columbia V1V1V7 , Canada) \\ (2 Institute of Applied Ecological Research, Chinese Academy of Sciences , Shenyang 110016, China)
}

\begin{abstract}
In-stream wood is defined as dead wood ( $>10 \mathrm{~cm}$ diameter and $>1 \mathrm{~m}$ length) in the stream channel. In-stream wood is a common, but important structural component in forest streams, particularly in relatively small creeks. Through review of research from the last 30 years (mainly from North America), we synthesize information on in-stream wood ecological functions (on channel morphology, nutrient cycling, sediment storage , aquatic habitat and biodiversity) , dynamics , spatial variation and relationship between in-stream wood (loadings and distribution) and disturbance (natural and human-caused). We also present wood management paradigms and future research directions.

Much research has demonstrated that in-stream wood has important ecological functions for aquatic ecosystems. In-stream wood can significantly change channel morphology through its role in intercepting sediments and water flow as well as stabilizing stream banks. Many aquatic habitat features such as pool , cover and substrate are positively related to in-stream wood characteristics. In-stream wood also affects nutrient cycling through its decomposition and interception of fine organic materials (e.g. , tree needles and branches). Because of these ecological functions, in-stream wood supports greater aquatic biodiversity and productivity. However, its ecological significance largely depends on types of forested watersheds and sizes of streams. As stream sizes increase, wood loading and its influence generally decrease. The size of individual wood pieces , however, increases with increasing stream size. In-stream wood also has large temporal variations or dynamics which are mainly driven by large-scale catastrophic forest disturbance (i.e. , fire, windthrow, etc). Understanding spatial and temporal variations and differences between natural and human disturbances is important for protecting and maintaining wood ecological functions. We provide suggestions for future in-stream wood ecological research in China.
\end{abstract}

Key words In-stream wood , Large Woody Debris (LWD) , Ecological functions , Spatial and temporal variability , Disturbance 
溪流倒木 (英文为 In-stream wood 或 Large Wood Debris (简称为 LWD) )，一般是指在河流内长度大于 $1 \mathrm{~m}$ 、直径大于 $10 \mathrm{~cm}$ 的木头。它在流域系统中常被 区分为两个相联结的过程:倒木进入河流的过程 (Recruitment)与在河流内发生的过程 (In-channel process)。前者是指河岸植被带的树木死亡之后或通过 各种方式(例如河岸冲涮、泥石流的作用)而进入河 流。这个过程是溪流倒木的来源, 它与森林植被(特 别是河岸植被)的状况、植被的干扰、地貌特征等有 直接的关系。倒木在河流内发生的过程是指在树木 进入河流后所发生的一系列过程, 它包括分解与破 碎化、储存、搬运、沉积等过程。人们对溪流倒木的 过程及其生态意义的认识是渐进的。例如在 20 世 纪 50 至 70 年代在美国的太平洋西北区及加拿大的 西部, 溪流倒木普遍被认为是对航运、鱼的洄游有阻 碍作用，因此所采取的措施是清除河流中的倒木。 后来发现这些措施造成河流系统结构简单化，水生 生境及生态功能退化等不良后果。由于这些问题的 出现, 美国、加拿大等一些发达国家自 20 世纪 80 年 代初就开始对溪流倒木的生态学进行了大量的研 究。这些研究表明, 溪流倒木对河流形态、泥沙拦 截、水生生境的形成、生物多样性、养分循环及碳循 环等许多方面都有重要的作用。并且基于这些研究 结果建立了林业与流域管理策略 那就是如何保护、 恢复及维持一定数量的溪流倒木, 以达到保持河流 生态系统的整体性。一些地区或部门甚至把溪流倒 木作为监测或者判别河流生态系统健康的指标之

虽然溪流倒木生态学的研究在一些发达国家在 近几十年来得到较大的重视, 但在中国有关这方面 的研究极少。溪流倒木的生态意义可能因森林类 型、流域的特点而异，但它既是流域生态系统(特别 是森林流域生态系统) 中的重要结构成分, 又是功能 过程之一,应该对它进行恰当的科学评价。对它的 研究既有科学理论上的意义, 又有实践上的应用价 值。本文的目的是综述国外 (主要是北美地区) 近 30 年来在溪流倒木生态学研究上所取得的主要成 果, 并阐述溪流倒木的生态管理措施及未来研究方 向, 为中国将来开展此方面的研究与管理提供一定 的参考。

\section{1 溪流倒木的生态重要性}

在论述溪流倒木的生态重要性之前,有必要介 绍一些与倒木有关的术语。倒木在河流中要么单独
个体存在, 要么以聚集体 $(\mathrm{Jam})$ 呈现。一般来讲, 溪 流倒木的生态作用与倒木或倒木的聚集体的大小有 关。较大的倒木或聚集体对河流形态的影响要比较 小的倒木或聚集体要大。溪流倒木对河流系统的影 响也与河流的大小有关。河流越大, 溪流倒木的影 响相对降低。因此，倒木对河流的影响或倒木的功 能与倒木的相对大小 (例如 倒木直径与河流宽度的 比例)有关。这也意味着在比较不同河流中的倒木 的影响时，用倒木的相对大小应该是更适当的指标。 表示倒木大小的参数量通常是直径与长度, 而倒木 存留量常用每 $100 \mathrm{~m}^{2}$ 河段的倒木体积或重量。河 流在流域系统中呈网络结构。源头或最小的支流被 称为 1 级河流, 2 个 1 级河流相汇而成的河流叫 2 级 河流。 3 级河流则是由 2 个 2 级支流相汇而成。依 此类推, 越大的河流其级别就越高。一般来讲 级别 较低的河流的坡度较大, 而级别较高的河流坡度较 小。

\section{1 倒木与河流形态}

倒木或倒木聚集体通过改变河流中的泥沙、水 流速度及河岸稳定性而影响河流形态。倒木对泥沙 具有明显的拦截作用，主要表现在 3 个方面:第一， 倒木本身可以拦截一部分泥沙; 第二, 倒木能够对水 流具有阻拦、分流的作用，从而形成流速较低泥沙沉 积区，第三，倒木具有稳固河岸及减低河岸冲涮的作 用，从而减低泥沙的形成与输送。倒木对泥沙的拦 截作用, 特别在较小的河流 (3 级或以下)中较大。 例如 Tally (1980) 估算森林溪流倒木在美国国家红 树公园河流中可拦截超过相当于 200 多年的河床滚 动泥沙总量。Montgomery 等 (2003) 总结了许多研 究, 认为倒木至少可拦截超过 10 倍于年泥沙量的数 目。Gomi 等(2001) 研究美国阿拉斯加州的 Maybeso 实验林及 Harris 流域中的倒木与泥沙储存的关系， 发现倒木的存留量与倒木后面的泥沙量呈显著的正 相关。有些研究者也从相反的角度来反证倒木对泥 沙的拦截(Bilby，1981; Smith et al.，1993)。这些研 究证实, 清除河流中的倒木会大量减少泥沙的拦截 量, 进而增加泥沙的输出。倒木对泥沙的拦截作用 使下游的泥沙减少, 也降低了河流中泥沙迁移、运输 的变异性。

倒木对泥沙的拦截作用因河流的大小而异。一 般来讲, 随着河宽或级别的增加, 倒木的稳定性降 低，因而其对泥沙的拦截作用也减低。在宽度较小 但坡度较大的河流中 (1 2 级), 倒木通常是形成深 潭 ( Pool) 的主要结构成分, 这种深潭有助于降低水 
的能量从而拦截更多的泥沙。Bilby 和 Ward (1989) 发现在宽度小于 $7 \mathrm{~m}$ 的河道中，有 $40 \%$ 的倒木对拦 截泥沙有作用, 而在宽度大于 $10 \mathrm{~m}$ 的河道中, 则只 有 $10 \%$ 的倒木对泥沙有影响。应该指出的是, 在坡 度较小、宽度较大的大河中 (大于 5 级) ,倒木对泥沙 拦截的作用是非常有限的。在一些特殊的情况下， 倒木还可能因为限制溢洪区或河道中河坝的形成， 从而增加泥沙的输出 (Nakamura \& Swanson, 1993)。

溪流倒木对河岸及河道的稳定、减少河岸的冲 涮具有重要的作用。在较小的河流中, 倒木(特别是 与水流方向垂直的倒木)易形成梯级 (Step) ,而在每 一个梯级下面往往是深潭, 这种梯级/深潭的结构能 降低或耗散水的冲涮能量，从而有助于河道的稳定 (Heede，1985a，1985b)。另一方面, 倒木对河岸具 有明显的加固作用，这种作用使得河岸的冲涮降低。 不少的研究证明, 清除倒木使河岸冲涮加重, 河道形 态发生改变(Beschta，1979；Adenlof \& Wohl，1994)。 根据在加拿大不列颠哥伦比亚省南部 Okanagan 流 域的森林溪流倒木的研究, Chen 等 (2005) 和 Scherer (2006) 发现有近 40\% 60\%的倒木对河岸的稳定性 有加固的作用。

\section{2 倒木与水生生境 (Aquatic habitat)}

倒木对水生生境的影响主要体现在对深潭形 成、河道基质构成及覆盖 (Cover) 等方面的影响。大 量的研究表明，倒木在坡度较大(>5\%)或中等 $(2 \%$ $\sim 5 \%)$ 的森林河流中，对于深潭的形成具有十分重 要作用(图 1)( Andrus et al. , 1988 ; Carlson et al. , 1990 ; Robison \& Beschta , 1990 ; Montgomery et al. , 1995 ; Abbe \& Montgomery, 1996 ; Beechie \& Sibley, 1997 ; Chen et al . 2006)。Bilby (1984) 研究美国华 盛顿州西南部森林小流域中发现，多于 $80 \%$ 的深潭 是由倒木形成的。Sedell 等 (1985) 在美国爱达荷州 的研究也得到类似的结论。根据 Lassettre 和 Harris (2001)的总结,在美国太平洋海岸坡度较大或中等 的森林溪流中，参与深潭形成的倒木的比例是 $20 \%$ ～80\%。Scherer 等 (2006) 和 Chen 等 (2006) 对加拿 大不列颠哥伦比亚省南部 Okanagan 流域的研究, 得 到近 $50 \%$ 的倒木参与深潭的形成。然而在坡度较 小的较宽河流中, 倒木对深潭形成的影响就有限, 且 深潭的类型主要是以 冲涮潭” (Scour pool) 为主 (Bilby \& Ward , 1989 , 1991 ; Robison \& Beschta , 1990 ; Richmond \& Fausch，1995)。这主要是在较宽的河流 中, 倒木的长度不能延伸至整个河宽, 对水流不能做 垂直拦截, 而由此所产生的潭则是 冲涮型”的。
倒木能对一些水生物种(如鱼)提供重要的掩蔽 场所(图 1)。特别是在进入河流的初期, 有相当多 的倒木附有大量枝条并悬在河道上, 这就为一些水 生物种提供了较掩蔽的场所（Bisson et al.，1987； Wondzell \& Bisson，2003)。倒木的这种覆盖作用随 着倒木或者枝条的不断分解、破碎化、冲湒，从而与 河道的直接接触增加而减小。

倒木对河道基质构成的影响使水生环境的多样 化被许多研究所证实(Bisson et al ., 1987 ; Buffington , 1998 ; Buffington \& Montgomery , 1999 ; Manga \& Kirchner , 2000 ; Gomi et al. , 2001)。根据 Montgomery 等(2003) 的总结, 倒木通过增加河床及河岸 的䊁粗度 (Roughness), 从而明显减少河床的切应力 (Shear stress) 及河道导流力 (Channel competence)。 其结果使河床的构成基质的平均直径减少 (Lisle， 1995 ; Buffington \& Montgomery , 1999)。另外, 倒木能 诱导河流中切应力与泥沙搬运力的变异性, 从而形 成具有不同基质的空间斑块（Montgomery et al. , 2003)。这两种作用( 降低河床构成颗粒的直径及增 加河床的空间异质性) 的综合影响使河流中水生生 境的多样性与可利用性增加。Buffington (1998) 发 现, 由于倒木的作用, 大马哈鱼产卵所需的砾石既使 在较陡、河床岩石较大的流域中也得到明显的增加。

\section{3 倒木与养分循环}

倒木在两个方面影响养分循环。其一是倒木本 身的养分的分解与释放; 其二是倒木通过截持或储 存影响养分的运输的时间及数量(Bilby，2003)。在 森林小河流系统中，上面两个过程对河流中养分的 动态都起着决定的作用。倒木主要含有 $\mathrm{C}$, 其它元 素的含量很低。倒木在水中的分解比在陆地要慢 (Harmon et al ., 1986 ; Scherer ,2004)。因此, 倒木在 漫长的分解过程中释放的养分较低。河流中除了倒 木以外, 还有一些有机碎屑 (叶子、枝等)。这些碎屑 虽然总量较低, 但所含的养分 (除 Ca 外)与倒木所含 的总量相接近 (Bilby，2003)。倒木通过截持或储存 有机碎屑影响养分循环, 这种作用可能比倒木本身 的养分循环更重要。在森林小溪流中，倒木或它的 聚集体由于能够降低水流流速与能量从而截持大量 从上游或河岸植物区输入的有机物碎屑。许多倒木 清除试验表明，在倒木从河道中移除后，有机碎屑和 养分输出明显增加 (Bilby \& Likens, 1980 ; Beschta， 1979)。相反，一些倒木增加试验表明，在倒木增加 后,河流中有机碎屑的储存量会增加。例如, Wallace 等 (1995) 在美国南阿巴拉契亚山森林小溪 

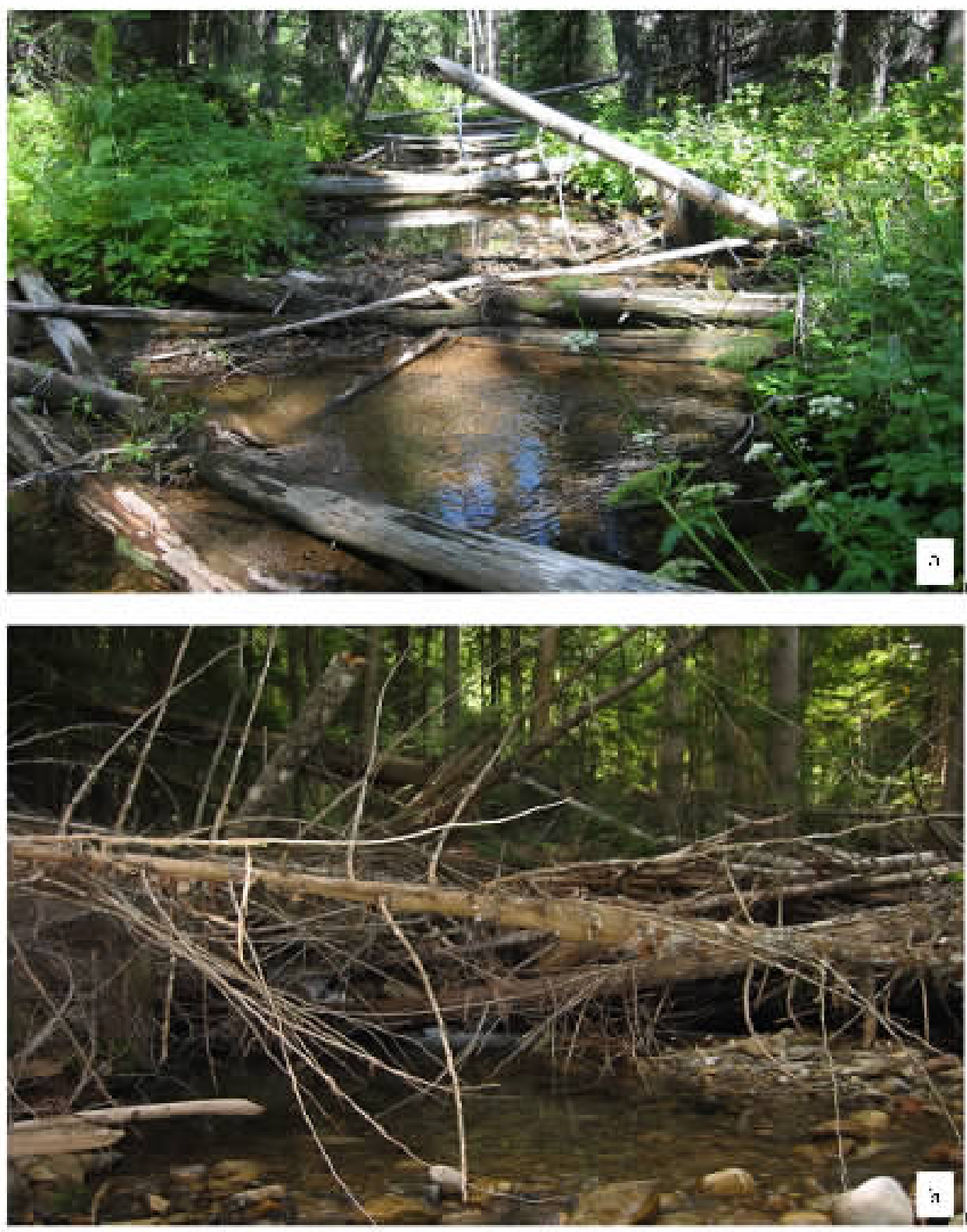

图 1 溪流倒木的生态功能(照片由魏晓华提供)

Fig.1 Ecological functions of in-stream wood (photos by Xiaohua Wei) $\mathrm{a}$ : 拦截作用及深潭的形成 Sediment interception and pool formation $\mathrm{b}$ : 提供掩蔽 Providing cover

流的研究表明,人为增加倒木使得被截持的有机碎 屑从 $88 \mathrm{~g} \mathrm{~m}^{-2}$ 增加到 $1568 \mathrm{~g} \mathrm{~m}^{-2}$ 。倒木对于养分 循环的影响，在较小的第一级或第二级河流中最明 显。随着河流宽度和级别的增加，倒木对养分的影 响则减少。这主要是在较大的河流中, 倒木的单位 面积的截留量要小,对有机碎首的截持作用也低。 根据 Bilby (2003)的资料, 当美国太平洋西北沿海地 区的河流宽度从 $5 \mathrm{~m}$ 增加到 $15 \mathrm{~m}$ 时, 通过倒木所截 持的养分含量下降 $80 \%$,而在新罕布什尔州的白 山 , 由倒木所截持的养分从第一级河流到第三级河 流下降了 10 倍之多 (Bilby , 1979)。倒木对大马哈鱼
的残体有明显的截持作用，这种截持作用对河流水 生系统养分及生产力的提高有十分明显的作用 (Bilby et al.,1998)。

$\mathrm{C}$ 循环的研究成为当今世界最重要的研究课题 之一。在 C 循环的研究中,一个挑战性的课题一直 困扰着科学家，那就是对全球的 $\mathrm{C}$ 收支估算达不到 平衡。这些不能平衡的 $\mathrm{C}$ 量被称为丢失的碳” (Missing carbon)。目前有一种假说是 :C 在流域系统 中可能通过输入、搬运、沉积、埋没等过程而最终未 能被测量与估算。美国现在已经在哥伦比亚流域开 展此方面的研究, 以此理解” 丢失的碳”。因此研究 
溪流倒木在流域系统中的输入、运输、沉积和埋没对 研究 C 循环具有重要的意义。倒木进入河流后, 经 历一系列过程包括分解、破碎、搬运等, 又由于其分 解较慢及河流中泥沙沉积, 有些倒木常部分或全部 被泥沙埋没或进入更大的系统 (湖泊、湿地或海洋) 而被沉淀。这些被水或泥沙埋没的倒木, 其分解非 常缓慢。根据 Guyette 等 (2002) 在美国密苏里州和 伊阿华州北部柇树林流域中的溪流倒木的研究, 因 倒木在水中或泥沙被埋没的环境中, 其存留时间可 能达 1.4 万年。

\section{4 倒木与生物多样性及生物产量}

溪流倒木对于生物多样性及产量有重要的影 响。第一, 倒木本身是河道中的一个重要的基质, 为 一些水生物种提供栖身或发展的场所; 第二, 倒木本 身含有养分, 通过分解、破碎化释放养分, 更重要的 是倒木可以拦截大量的有机碎屑, 这就为河流生态 系统提供了能量与物质, 第三, 倒木改变了河流的形 态, 产生更多适合不同物种的生境。

倒木在河流中, 因分解较慢, 在河流中滞留的时 间较长。又由于有不断的倒木从上游或河岸带输 入，从而在一个河段中常有处于不同分解阶段的倒 木, 这种倒木可以单独或呈聚集状态, 也可有不同的 排列方向。倒木的这种分布就产生了河段基质的多 样化或河段的异质化。这种异质化能够增加一些物 种的栖身或发展的场所。特别在坡度较小含有大量 细沙的河流，倒木则成为稳定的、质地很硬的河流基 质,成为一些依赖倒木居住的物种的重要场所 (Benke \& Wallace , 2003 ; Smock et al. 1989)。另外， 倒木的表面由于长期的分解及水的冲击, 其表面随 着时间的增长而复杂化。这种由于分解而产生的微 生境的多样化从而使大型无脊椎动物群体的多样性 增加 (Hax \& Golladay, 1993 ; Magoulick，1998)。 O' connor (1991)也发现表面粗粘的倒木具有更高的 大型无脊椎动物群体生物多样性。

倒木能有效拦截大量的有机碎屑 (枝条、叶子 等) , 这些有机物质是许多水生物种的重要物质与能 量(Smock et al.,1989; Casas，1997)。因此, 倒木通 过影响河流系统中的养分与能量结构而对水生生物 的生产力与多样性有提高的作用 (Wallace et al. , 1996)。另外，由于倒木能有效控制河流网络中泥沙 的产生、搬运及分布格局而影响生物多样性。 Palmer 等(1996)发现, 倒木能够产生水速较小、基质 较细的沉积区, 从而为一些物种在洪水时提供了避 难所。有些沉积区由于泥沙的不断累积而变成坝,
甚至成为植被岛 (Ward et al. , 1999)。倒木还可以 通过截流泥沙而改变河道纵向的坡度, 这种改变有 助于河流与浅层地下水的交换，而这种交换有助于 水温与水质的调节 (Harvey \& Bencana，1993)。因 此, 倒木可以控制河流网络中泥沙及有机物质的储 存与分布，从而增加水生生境的复杂性而影响生物 的多样性。

更重要的是倒木通过对河流形态的改变而影响 生物的多样性与生产力。有关这方面的研究较多, 特别是倒木对于鱼类种群的影响。正如前面所述， 倒木在各个尺度上 ( 从倒木个体的周围小尺度到整 个河流网络的大尺度)对河流形态有影响 (尽管这种 影响因尺度的增加而降低)。这种影响使生境的复 杂性增加。而复杂的生境则有助于生物多样性的维 持与提高 (Hicks et al., 1991 ; Reeves et al., 1993; Shields et al . , 1994)。然而, 也有一些研究发现, 倒 木并不能增加生物多样性 (Chen, 1999) , 个别研究 甚至发现倒木有降低大型无脊椎动物生物多样性的 结论 (Murphy \& Hall , 1981)。不过, 在他们研究的系 统中光照是影响生产力的主要因素, 而森林采伐(减 少倒木) 则可以提高光照及生产力, 进而影响生物多 样性。

影响河流生态系统生物多样性的因素很多。倒 木及其形成的生境只是其中的一个部分因素。其它 因素包括水温、水量、基质构成、干扰因素、河岸带植 被特征等, 所有这些因素综合决定着生物多样性与 生产力。事实上, 很难单独区分倒木及其它因素对 生物多样性及生产力的影响。

\section{2 倒木的时空变异性}

溪流倒木进入河流的过程可细分为 4 个:1) 河 岸植被带中单株树木由于竞争或者病虫害引起的死 亡 2)大规模的森林火灾、病虫害或人为干扰 ; 3 河 岸植物由于冲湒或泥石流所输入的树木 ;4)上游倒 木的输入。倒木进入河道内发生的过程则包括为分 解、破碎化、运输、沉积或埋没等。在任一时间、任一 河段内的倒木存留量及其分布取决于上面所有过程 的综合作用。由于这些过程, 特别是倒木的输入过 程具有较大的随机与不确定性, 这就导致溪流倒木 具有很大的时空变异性 (Swanson, 2003; Wei, 2003)。

\section{1 溪流倒木的空间变异性}

河道中倒木的存留量由于受许多因素 (河岸植 被、干扰、水文、河流形态等)的影响，在空间尺度上 
呈现很大的变异性 (Bisson et al., 1987 ; Abbe \& Montgomery，1996，2003)。这种变异性既表现在同 一尺度的河道之间，也存在于不同尺度的河道之间。 在同一尺度的河道之间，虽然河流的大小一致或都 属于同一等级的河流, 但由于河流的坡度差异, 河岸 植被受干扰的历史不同, 其倒木载量的差异就可能 较大。例如 根据对加拿大不列颠哥伦比亚省南部 Okanagan 流域的调查, 虽然河流都是二级, 且植被都 是较成熟的小干松林 (Pinus contorta ssp. latifolia Engelm. ex S. Wats.) ,但河道中的倒木存留量相差 数倍以上 (Chen et al. ,2005)。Harmon 等 (1986)比 较未受干扰的不同温带森林中的溪流倒木存留量， 发现森林类型不同, 其倒木载量相差很大(红木林 > $1000 \mathrm{~m}^{3} \mathrm{hm}^{-2}$, 针叶林 $200 \sim 1000 \mathrm{~m}^{3} \mathrm{hm}^{-2}$; 阔叶 林 $<200 \mathrm{~m}^{3} \mathrm{hm}^{-2}$ )。在不同尺度的河道之间，随着 河流宽度的增加或河流从低级到高级,倒木的存留 量一般减少。这主要是在较大河流中由于倒木的输 入减少，且搬运能力增加所致 (Naiman et al ., 2002 ; Swanson, 2003)。尽管这个结论是普遍认可的, 但仍 有一些稍不同的结论, 例如, Chen 等(2005)发现溪流 倒木在加拿大不列颠哥伦比亚省的 Okanagan 流域 的二级河流中的存留量比一级河流要大。尽管如 此, 它们的存留量都要高于更高级或更大的河流, 这 又与普遍认可的结论相吻合:即随着河流级别的增 加, 倒木的存留量会减少。

倒木的搬运机制与聚集方式也有空间变异。在 宽度较小坡度较大的 1 或 2 级河流中, 由降雨或洪 水诱发的碎石流是搬运倒木的主要机制 (Keller \& Swanson , 1979 ; Nakamura \& Swanson , 1993)。而在中 级或高级的大河流中，倒木的漂运则是倒木搬运的 主要机制 (Swanson et al. , 1976 ; Keller \& Swanson , 1979)。倒木聚集方式的空间差异主要表现在, 随着 河流级别增加，倒木则从随机排列的单个个体为主 变为以大的、有组织的聚集体为主 (Montogomery et $a l$ ，2003)。这主要是由于在较小的河流中，倒木的 长度往往大于河流宽度, 倒木的稳定性高, 从而不易 被搬移。因此, 倒木多数是倒在哪里就相对固定在 哪里。而在大河流中, 由于倒木的稳定性差, 被搬运 的能力较强, 许多倒木甚至被漂运, 这些倒木可在一 些特定的地方成为聚集体 (Gurnell et al.，2002; Abbe \& Montgomery，2003)。一般来讲，经过越多搬 运的倒木而形成的聚集体, 其紧实程度就越高 (Swanson , 2003)。

\section{2 溪流倒木的动态性或时间变异性}

溪流中的倒木受水流冲击与搬运, 又与泥沙及 河道相互作用, 它在河流网络系统呈现独特的分布。 有的全部或部分在水中，有的可能被埋没，也有可能 在深水中。这种分布在较大程度上决定了倒木在河 流中存留的时间。根据 Chen 等 (2006) 在加拿大不 列颠哥伦比亚省南部 Okanagan 流域中对溪流倒木 的研究, 大部分倒木因其部分时间在水中 部分时间 在空中, 故其分解相对要快, 它们的存留时间大约在 $100 \sim 150$ 年左右。而有一部分倒木由于搬运的结 果，终年在水底中，其存留时间在 $400 \sim 600$ 年左右； 还有一部分可能被泥沙埋没, 其存留时间应更长。

倒木在河道中其存留量的动态性与倒木的收支 量是密不可分的。倒木的各种来源决定了某一河流 倒木的输入或收入, 而倒木的分解、河流搬运则决定 了其支出 (Benda et al. ,2003)。在任一时间内，倒 木的存留量是其收入与支出的平衡的结果。在森林 河流生态系统中, 森林毁灭性的自然干扰 (如火灾) 往往产生大量倒木输入, 这种输入过程可持续几十 年。在载量达到最高值后, 倒木的动态变化则取决 于搬运、分解及由更新植被中由于竞争而死亡的倒 木输入。根据这些过程的综合及模拟, 研究者已提 出了森林流域中溪流倒木的一般动态模式 (Mishall et al . , 1989 ; Bragg , 2000 ; Scherer et al .,2006)(图 2 , 图 3)。Wei (2003) 认为在一个由火烧作为主要 自然干扰的森林流域系统中，森林毁灭性的火烧每 隔一段时间便发生, 而由数次火烧所产生及驱动的 溪流倒木动态可用波动来表达 (图 2)。这种由波动 表达的倒木变化可能是倒木在更长时间尺度的动态 变化规律。

研究倒木的时空动态规律是一个前沿性的科学 难题。尽管在过去 30 多年内, 对溪流倒木的研究受 到广泛的重视, 但绝大多数的研究局限河段 (Reach) 尺度或较小尺度系统，只有非常少的研究考虑倒木 在整个河流网络中的输入、搬运、沉积等规律 (Swanson , 2003)。在倒木动态方面, 绝大多数的研 究所用的时间尺度较短。即使有少数的研究考虑长 期的动态规律, 但他们主要是以计算机模型来进行 的, 由实测数据而组建的长期倒木动态模型几乎没 有。Scherer 等(2006) 利用空间代时间方法, 应用野 外资料组建了在加拿大不列颠哥伦比亚省南部 Okanagan 流域的小干松森林流域倒木的动态模型。 Swanson(2003) 提出将来要从景观角度或整个流域角 


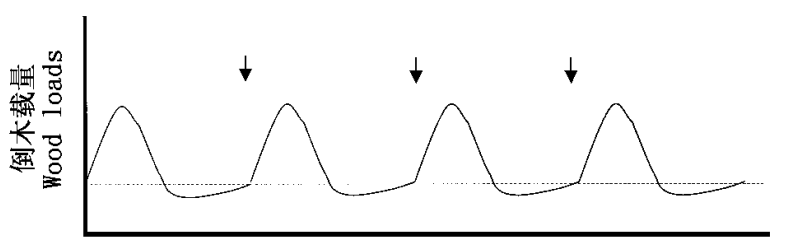

时间 Time

图 2 假设的倒木的动态波动格局 :由数次火烧所产生及驱动的 溪流倒木长时间尺度的动态变化规律

Fig.2 Assumed pulse pattern of in-stream wood dynamics : driven by multiple catastrophic wildfire disturbance events over a long period

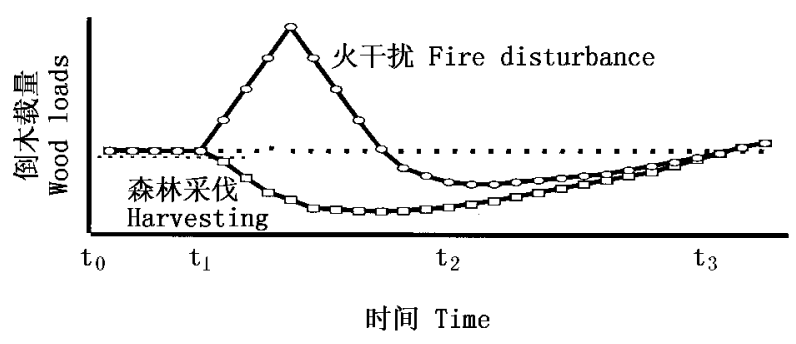

图 3 森林火灾与森林采伐对溪流倒木存留量的动态变化的 影响的一般溪流倒木动态模式(Bragg，2000)

Fig. 3 Difference in long-term dynamics of in-stream wood loadings between wildfire disturbance and timber harvesting (cited from Bragg (2000) with author' s permission)

$t_{1}$ : 第 50 年 The fiftieth year $t_{2}$ : 第 150 年 One hundred fiftieth year $t_{3}:$ 第 300 年 Three hundreds year

度来研究倒木的特点及其生态问题，既考虑倒木的 空间尺度又考虑倒木的时间尺度，从而达到认识倒 木在景观或整个流域的特点及生态意义的目的。

\section{3 干扰与倒木}

有关干扰的定义较多, 目前引用较多的是 Pickett 和 White (1985) 的定义, 即干扰是在时间尺度上 任何能中断生态系统、群落的结构与过程并改变资 源的分布及物理生境的相对间断的事件。我们一般 用频度、强度和类型来描述、表达干扰。例如，在描 述森林火的干扰时, 火的类型是指林冠火、地表火 等; 火的频度则指火出现的概率或多少年一遇; 火的 强度是指火燃烧的程度。从干扰的起因来讲,干扰 可划分为自然干扰和人为干扰。自然干扰在森林流 域生态系统中包括发生森林中的火、风倒和病虫害 等及发生于河流中的岩屑流、泥石流、大洪水和河流 冲涮等。人为干扰常包括森林采伐、筑路、土地利用 的改变、河道工程化和水坝等。千扰对溪流倒木的 输入、运输及在河流网络中的分配具有决定性的作 用。要研究和认识倒木在河流中的特点及其生态意
义 就必须研究干扰与倒木的关系。

\section{1 自然干扰与倒木}

大规模、毁灭性的森林干扰可为河流提供大量 倒木。Minshall 等(1989) 研究美国黄石公园 1988 年 火灾对倒木输入河流的影响，发现河流中倒木在火 灾后有明显的增加。Chen 等(2005)对加拿大不列颠 哥伦比亚省的南部 Okanagan 流域中的研究发现在 火烧后的河流中的倒木即使在 $80 \sim 100$ 年后, 其存 留量仍高于火烧之前的河流中。火除了直接影响倒 木的输入外，还影响倒木的搬运过程。森林火灾后， 由于森林失去了覆盖, 以及土壤疏水使地表径流、水 土流失增加，也使泥石流及水灾的概率与强度都相 应加大，从而增大倒木被搬运的能力及泥石流的形 成。

其它自然干扰，例如 病虫害、风倒、泥石流等也 对倒木及其分布产生深刻影响 (Gurnell et al. , 2002 ; Nakamura \& Swanson, 2003)。Lienkaemper 和 Swanson(1987) 观察到大约 69\%的溪流倒木是由于 风倒引起的。在日本登陆的台风也往往产生大量的 风倒而使河流中的倒木明显增加 ( Nakamura \& Swanson, 2003)。

特别指出的是, 自然干扰可单独对森林流域系 统起作用, 但更常见的是它们之间的联合作用。例 如森林风倒后, 泥石流或岩屑流常被诱发。又比方 在加拿大不列颠哥伦比亚省的内陆的小干松森林流 域中, 天牛常常大面积感染森林, 而死亡后的树木为 森林火灾提供了大量的可燃物质。火灾以后通常又 能进一步诱发泥石流。因此, 在该地区, 病虫害、火 灾及泥石流是综合起来对流域进行干扰的。这些干 扰是倒木动态变化的来源与动力。

\section{2 人为干扰与倒木}

森林经营, 特别是河岸植被的采伐和林道的修 筑对倒木的来源具有非常严重的负面影响。河岸植 被采伐、运走后，倒木的陆地来源几乎失去，这种情 况可持续相当长的时间直至新的河岸植被恢复成 林，并产生新的死木进入河流。由于缺乏倒木的来 源 故河道中的倒木存留量会逐渐减少直至河岸植 被的恢复(假设上游流入的倒木等于该河段流失的 倒木) (Bilby \& Ward , 1991；Hogan et al., 1998 ; Wei , 2003)。森林采伐还由于改变水文与水土流失 而诱发更大更多的泥石流、岩首流从而增加倒木的 搬运, 使河段中的倒木存留量进一步减少。

其它人为干扰方式包括林地农业化、河道的工 程化等都有降低溪流倒木存留量的作用。这是由于 
这些人为干扰能降低倒木的来源及增大倒木的搬运 能力的综合作用所致。水坝 (水库)或泥沙沉淀池塘 的修建，由于降低、阻拦河道对倒木的搬运能力, 则 对倒木具有截持与存留的作用。不管是哪种人类干 扰方式, 对倒木存留量及其分布都具有明显的作用。 特别是在一些经历城市化、农业化及林业强度化的 河道中，倒木作为河道生态系统的一个重要的结构 物质几乎消失。这种结构上的消失必然造成河流水 生系统的功能的退化。

\section{3 自然干扰与人为干扰的区别}

自然干扰与人为干扰的区别在于:1)干扰的方 式 2)干扰的影响或结果。对于干扰的方式而言，自 然干扰常常是随机的,干扰的频度与强度有较大的 变异, 而人类干扰则是确定性的, 常有固定一致的频 度与强度。仔细比较一下森林自然火烧与人类森林 采伐就不难理解上面的差异。在干扰的影响 (结果) 方面，自然干扰与人为干扰也有明显区别，而这种差 别与干扰方式的差别是有关的。

研究自然干扰与人类干扰的区别已受到越来越 多的重视。这是由于 第一, 人们逐渐认识到自然干 扰是生态系统中的一部分, 具有重要的生态意义, 系 统的功能与整体性与干扰密不可分。生态系统就是 在这种干扰一恢复一再干扰一再恢复的循环中进化 的。第二, 由于人口急增, 人为干扰正在逐渐取代自 然干扰而成为主要干扰方式, 而人为干扰的生态后 果使人们有理由担心生态系统的持续性。鉴于此， 目前有一种较现代的提法就是把模拟自然过程、自 然干扰作为我们设计人为干扰的指南。尽管人为干 扰不可能完全复制与模拟自然干扰, 但在设计不可 避免的人为干扰时, 考虑或尊重一些重要的自然过 程应该是理性与科学的选择。而要做到这一点, 就 必须理解与研究自然干扰与人为干扰的区别。

有关比较自然干扰与人为干扰对溪流倒木影响 的研究很少。Bragg (2000) 用模型的手段比较森林火 灾与森林采伐对溪流倒木存留量的动态变化的影响 (图 3)。Chen 等(2005) 和 Scherer 等 (2006) 则用空间 代时间的方法组建倒木的时间动态规律, 比较火灾 与采伐的区别。

\section{4 溪流倒木的生态管理与未来的研究方向}

\section{1 倒木的生态管理模式}

由于近 30 年对溪流倒木的研究，对其在河流生 态系统及河岸植被生态的作用在北美 (特别是美国 西部及加拿大不列颠哥伦比亚省)有不断的认识，从
而导致倒木管理模式的变化。例如在 20 世纪初、中 期的美国太平洋西北地区, 由于当时人们普遍认为 溪流中倒木对鱼的洄游具有阻碍作用, 因此采取的 管理模式是清除河流中的倒木。但后来发现大量清 除河段中的倒木导致鱼的水生生境的退化。又由于 大量的科学研究证实了倒木的生态作用，从而在 20 世纪 80 至 90 年代采用的倒木管理模式是通过保护 河岸植被带从而维持河流倒木的来源并在退化的河 流中人工引入倒木。这种模式的核心是维持一定的 倒木数量。实际上, 溪流倒木已被许多地区作为一 个重要的河流生态系统健康指标来监测与管理。虽 然这种模式对于保护河流水生生态系统有很好的指 导意义, 但一个最根本的问题并没有解决, 即对一个 河段来说到底多少倒木存留量才能适当地维持河流 水生生态系统的生态功能? 由于溪流中的倒木存留 量具有很大的空间、时间变异性, 这就决定了我们不 可能用一个准确的特定值来作为倒木的适当量, 也 许一个变化范围更合适 (Lisle, 2002 ; Wei , 2003)。 虽然一个新的倒木管理模式还没有出现, Wei(2003) 建议未来的倒木管理模式应该考虑以下几个方面： 1)溪流倒木空间的巨大变异性, 这也意味着倒木的 适当量” 应是因地而异 2) 倒木的时间动态性, 并把 干扰作为动态性的一部分来研究;3) 河流的整个网 络结构与倒木的关系。

保护或恢复河岸植被带对保护河流系统来讲是 一项有效的林业措施而被许多地区采用。例如在加 拿大不列颠哥伦比亚省, 林业政策就规定一定宽度 的河岸植被带必须保留着, 而它的宽度取决于河道 的宽度 (British Columbia Ministry of Forests , 1995)。 然而, 这种政策主要是基于河岸植被对控制泥沙、水 温、水质、食物与能量方面考虑的, 并没有考虑河岸 植被带对倒木来源的重要性。这种政策的后果是， 当河岸植被带被自然干扰 (例如病虫害) 后, 往往被 采伐利用, 因为人们普遍认为河岸植被死亡后, 它对 水文、水质、水温和泥沙等的调节功能已不存在。但 事实上, 河岸植被被自然干扰后, 它能为河流提供大 量的倒木。另外一个争论较多的问题是被保留下的 河岸植被带如果其宽度较窄, 能否诱发更多更大的 风倒，而影响倒木的输入过程。因此，在设计保护河 岸植被带的策略时,也应考虑它对溪流倒木的动态 性。

\section{2 末来的研究方向}

在过去几十年内, 有关倒木方面的研究受到了 广泛的重视, 但绝大部分的研究局限在空间较小的 
河段及时间较短的尺度, 未来的研究方向应该在下 面几个方面 :

1）空间尺度 例如倒木在整个河流网络中的输 入、运输、分布的规律; 倒木及其生态作用在不同空 间尺度上的调控过程与作用, 评价在不同空间尺度 上河岸植被及其生态环境与溪流倒木的相互作用。

2) 时间尺度: 从模拟与野外调查相结合的角度 研究溪流倒木的长期动态性; 并结合空间尺度研究 倒木的景观格局、过程及调控因素。

3）把溪流倒木生态的研究置于干扰的框架之 中。研究不同自然干扰 (火灾、风倒、病虫害等)对倒 木的影响; 倒木在自然环境下的变异度; 人为干扰 (森林采伐、土地利用等) 对倒木的输入、搬运、沉淀 等过程的影响;并进一步研究自然干扰与人为干扰 在倒木及其有关的生态过程的差异。

4) 溪流倒木的生态恢复问题: 在被人为干扰而 导致溪流倒木缺乏，水生生境退化的系统，如何维持 适当的倒木存留量? 而适当的倒木存留量又是多 少?

\section{3 对中国开展溪流倒木生态研究的建议}

中国具有世界上最完善的植被系统, 这就为中 国研究与比较不同流域的植被类型的倒木生态提供 了一个独特的机会。中国在溪流倒木生态方面的研 究目前基本上是空白, 重视不够。这可能有两方面 的原因 : 其一是对倒木的生态作用认识不够 ; 其二, 由于中国是一个缺水且水污染严重的国家，对流域 中的水量与水质的关注远高于对其它流域过程的重 视。

但随着中国经济不断发展, 对环境的不断重视, 保护河流水生生态系统的整体性及健康必将成为重 要的研究与管理课题。同时对溪流倒木的研究也有 助于探讨水生生物多样性、全球气候变化与碳平衡、 陆地系统与水生生态系统相互作用等全球范围的热 点课题。

由于溪流倒木在河流系统中呈现很大的变异 性, 其生态作用也因河流的大小 (级别)、植被类型与 干扰的历史、河流中的目标资源而异。北美开展倒 木的研究最早是从保护大马哈鱼的需要开始的, 现 在发展到以对整个水生生态系统的保护为目的。可 以肯定, 倒木在中国众多且差异很大的流域系统中 的生态作用是不同的。因此, 中国开展溪流倒木生 态研究的第一步是把重点放在倒木对目标资源或生 态问题有重要关系的森林河流中。同时, 应把研究 倒木生态与水生生物多样性的保护、碳循环与平衡、
水土流域与河流泥沙的控制等重要生态问题结合起 来。

\section{5 结 论}

溪流倒木是自然河流生态系统重要的结构组成 部分，它对泥沙、养分的截留、河流形态的改变、水生 与河岸植被生境的形成及生物多样性等具有重要的 生态意义。然而, 它的生态功能因河流的特征 (大 小、坡度等)、植被类型与干扰、河流中的目标资源不 同而异。溪流倒木与其有关的生态功能在空间与时 间上具有很大的变异性, 而在这方面的研究较少, 是 未来研究的重点。人为干扰 (森林采伐、土地利用 等)影响倒木的来源及在河流中的搬运过程而降低 倒木的存留量及其所相关的生态作用。研究人类干 扰、自然干扰及其倒木生态的不同影响有助于我们 正确理解倒木在河流网络中的动态, 也有助于我们 设计河流倒木的保护措施。

\section{参 考 文 献}

Abbe TB, Montgomery DR (1996). Large woody debris jams, channel hydraulics and habitat formation in large rivers. Regulated Rivers Research and Management, 12, 210-221.

Abbe TB, Montgomery DR (2003). Patterns and process of wood debris accumulation in the Queets Rivers basin, Washington. Geomorphology, 51, 81- 107.

Adenlof KA, Wohl EE (1994). Controls on bedload movement in a subalpine stream of the Colorado Rocky-Mountains, USA. Artic and Alpine Research, 26, 77 - 85 .

Andrus CW, Long BA, Froehlich HA (1988). Woody debris and its contribution to pool formation in a coastal stream 50 years after logging. Canadian Journal of Fisheries and Aquatic Management, 45, $2080-2086$.

Beechie TJ, Sibley TH (1997). Relationships between channel characteristics, woody debris, and fish habitat in northwestern Washington streams. Transactions of the American Fisheries Society, 126, 217-229.

Benda L, Miller D, Sias J, Martin D, Buffington JM (2003). Wood recruitment processes and wood budgeting. In: Gregory SV, Boyer KL, Gurnell AM eds. The Ecology and Management of Wood in World Rivers. American Fisheries Society, Symposium 37, Bethesda, Maryland. 49-73.

Benke AC, Wallace JB (2003). Influence of wood on invertebrate communities in streams and rivers. In: Gregory SV, Boyer KL, Gurnell AM eds. The Ecology and Management of Wood in World Rivers. American Fisheries Society, Symposium 37, Bethesda, Maryland. 149 - 177.

Beschta RL (1979) . Debris removal and its effects on sedimentation in an Oregon Coast Range stream. Northwest Science, 53, 71 - 
77 .

Bilby RE (2003) . Decomposition and nutrient dynamics of wood in streams and rivers. In: Gregory SV, Boyer KL, Gurnell AM eds. The Ecology and Management of Wood in World Rivers. American Fisheries Society, Symposium 37, Bethesda, Maryland. $135-147$

Bilby RE, Fransen BR, Bisson PA, Walter JK (1998). Response of juvenile coho salmon and steelhead to the addition of salmon carcasses to two streams in southwest Washington, USA. Canadian Journal of Fisheries and Aquatic Science, 55, 1909 - 1918.

Bilby RE (1981). Role of organic debris dams in regulating the export of dissolved organic and particulate matter from a forested watershed. Ecology, 62, $1234-1243$.

Bilby RE (1984). Removal of woody debris may affect stream channel stability. Journal of Forestry, 82, 609-613.

Bilby RE (1979). The Function and Distribution of Organic Debris Dams in Forest Stream Ecosystems. PhD dissertation. Cornell University, Ithaca, New York.

Bilby RE, Likens GE (1980). Importance of organic debris dams in the structure and function of stream ecosystems. Ecology, 61, $1107-1113$.

Bilby RE, Ward JW (1989) . Changes in characteristics and function of woody debris with increasing size of streams in western Washington. Transactions of the American Fisheries Society, 118, $368-378$

Bilby RE, Ward JW (1991). Characteristics and function of large woody debris in streams draining old growth, clear-cut, and second-growth forests in southwestern Washington. Canadian Journal of Fisheries and Aquatic Sciences, 48, 2499 - 2508.

Bisson PA, Bilby RE, Bryant MD, Dolloff CA, Grette GB, House RA, Murphy ML, Koski KV, Sedell JR (1987). Large woody debris in forested streams in the Pacific Northwest: past, present, and future. In: Salo EO, Cundy TW eds. Streamside Management: Forestry and Fishery Interactions. Institute of Forest Resources, University of Washington, Seattle, Washington, 143 190 .

Bragg DC (2000). Simulating catastrophic and individualistic large wood debris recruitment for a small riparian system. Ecology, $81,1383-1394$

British Columbia Ministry of Forests (1995). Riparian Management Area Guidebook, Forest Practice Code. Victoria, British Columbia.

Buffington JM (1998). The Use of Streambed Texture to Interpret Physical and Biological Conditions at Watershed, Reach and Subreach Scales. $\mathrm{PhD}$ dissertation. University of Washington, Seattle.

Buffington JM, Montgomery DR (1999) . Effects of hydraulic roughness on surface textures of gravelbed rivers. Water Resources Research, 35, $3507-3522$.

Carlson JY, Andrus CW, Froehlich HA (1990). Woody debris, channel features, and macroinvertebrates of streams with logged and undisturbed riparian timber in northeastern Oregon, USA. Canadian Journal of Fisheries and Aquatic Sciences, 47, 1103 1111 .

Casas JJ (1997) . Invertebrate assemblages associated with plant debris in a backwater of a mountain stream: natural leaf packs vs. debris dam. Journal of Freshwater Ecology, 12, 39-49.

Chen GK (1999). The Relationship Between Stream Habitat Complexity and Anadromous Salmonid Diversity and Habitat Selection. $\mathrm{PhD}$ dissertation, Oregon State University, Corvallis.

Chen X, Wei X, Scherer RA, Luider C, Darlington W (2006) . A watershed scale assessment of in-stream large woody debris patterns in the southern interior of British Columbia. Forest Ecology and Management, 229, 50-62.

Chen X, Wei X, Scherer RA (2005). Influence of wildfire and harvest on biomass, carbon pool and decomposition of large woody debris in forested streams of southern interior British Columbia. Forest Ecology and Management, 208,101 - 114 .

Gomi T, Sidle RC, Bryant MD, Woodsmith RD (2001). The characteristics of woody debris and sediment distribution in headwater streams, southeastern Alaska. Canadian Journal of Forest Research, $31,1386-1399$.

Gurnell AM, Piègay H, Swanson FJ, Gregory SV (2002) . Large wood and fluvial processes. Freshwater Biology, 47, 601-619. Guyette, RP, Cole WG, Dey DC, Muzkia RM (2002). Perspectives on the age and distribution of large wood in riparian carbon pools. Canadian Journal of Fisheries and Aquatic Sciences, 59, $578-585$.

Harmon ME, Franklin JF, Swanson FJ, Sollins P, Gregory SV, Lattin JD, Anderson NH, Cline SP, Aumen NG, Sedell JR, Lienkamper JW (1986) . Ecology of coarse woody debris in temperate ecosystems. In: MacFadyen A, Ford ED eds. Advances in Ecological Research. Academic Press, London, 133 - 302.

Harvey JW, Bencala KE (1993). The effects of streambed topography on surface-subsurface water exchange in mountain catchments. Water Resources Research, 29, 89 - 98.

Hax CL, Golladay SW (1993). Macroinvertebrate colonization and biofilm development and leaves and wood in a boreal river. Freshwater Biology, 29, $79-87$.

Heede BH (1985a). Channel adjustments to the removal of log steps: and experiment in a mountain stream. Environmental Management, 9, 427 - 432 .

Heede BH (1985b) . Interactions between streamside vegetation and stream dynamics. In: Proceedings-Riparian ecosystems and their management: Reconciling contlicting uses. USDA Forest Service, General Technical Report, RM-120. 54 - 58 .

Hicks BJ, Hall JD, Bisson PA, Sedell JR (1991). Responses of salmonids to habitat changes. In: Meehan WR ed. Influence of Forest and Rangeland Management on Salmonid Fishes and Their Habitat. American Fisheries Society, Special Publication 19, Bethesda, Maryland. 483 - 518 .

Hogan DL, Bird SA, Hassan MA (1998). Spatial and temporal 
evolution of small coastal gravel-bed streams: influence of forest management on channel morphology and fish habitats. Gravel-Bed Rivers in the Environment. In: Klingeman PC, Beschta RL, Komar PD, Bradley JB eds. Highlands Ranch, Colorado. Water Resources Publications, USA. $1701-1720$.

Keller EA, Swanson FJ (1979) . Effects of large organic material on channel form and fluvial processes. Earth Surface Processes, 4, $361-380$.

Lassettre NS, Harris RR (2001). The geomorphic and ecological influence of large woody debris in streams and rivers. Center for Forestry Report, University of California at Berkeley. http:// frap. cdf. ca. gov/publications/lwd/LWD-paper. pdf.

Lienkaemper GW, Swanson FJ (1987). Dynamics of large woody debris in streams in old-growth Douglas-fir forests. Canadian Journal of Forest Research, 17, 150 - 156.

Lisle TE (2002). How much dead wood in stream channels is enough? USDA Forest Service General Technology Research, PSWGTR-181, 85 - 93.

Lisle TE (1995). Effects of coarse woody debris and its removal on a channel affected by the 1980 eruption of Mount St. Helens, Washington. Water Resources Research, 31, 1797 - 1808 .

Magoulick DD (1998). Effects of wood hardness, condition, texture and substrate type on community structure of stream invertebrates. American Midland Naturalist, 139, 187 - 200.

Manga M, Kirchner JW (2000). Stress partitioning in streams by large woody debris. Water Resources Research, 36, 2373 - 2379.

Minshall GW, Brock JT, Varley JD (1989). Wildfires and Yellowstone's stream ecosystems. BioScience, 39, $707-715$.

Montgomery DR, Collins BD, Buffington JM, Abbe TB (2003). Geomorphic effects of wood in rivers. In: Gregory SV, Boyer KL Gurnell AM eds. The Ecology and Management of Wood in World Rivers. American Fisheries Society, Symposium 37, Bethesda, Maryland, $21-47$.

Montgomery DR, Abbe TB, Buffington JM, Peterson NP, Schmidt KM, Stock JD ( 1996). Distribution of bedrock and alluvial channels in forested mountain drainage basins. Nature, 381, 587 -589 .

Montgomery DR, Buffington JM, Smith RD, Schmidt KM, Pess G. (1995). Pool spacing in forest channels. Water Resources Research, 31 .

Murphy ML, Hall JD (1981) . Varied effects of clear-cut logging on predators and their habitat in small streams of the Cascade mountains, Oregon. Canadian Journal of Fisheries and Aquatic Sciences, 38, $137-145$.

Naiman RJ, Balian EV, Bartz KK, Bilby RE, Latterell JJ (2002). Dead wood dynamics in stream ecosystems. USDA Forest Service General Technology Research, PSW-GTR-181, 23 - 48.

Nakamura F, Swanson FJ (2003). Dynamics of wood in rivers in the context of ecological disturbance. In: Gregory SV, Boyer KL Gurnell AM eds. The Ecology and Management of Wood in World Rivers. American Fisheries Society, Symposium 37, Bethesda,
Maryland, $279-297$.

Nakamura FS, Swanson FJ (1993). Effects of coarse woody debris on morphology and sediment storage of a mountain stream system in western Oregon. Earth Surface Processes and Landforms, 18, $43-61$.

O' connor NA ( 1991$)$. The effects of habitat complexity on the macroinvertebrates colonizing wood substrates in a lowland stream. Oecologia, 85, 504-512.

Palmer MA, Arensburger P, Martin AP, Denman DW (1996). Distribution and patch specific responses: the interactive effects of woody debris and floods on lotic invertebrates. Oecologia, 105, $247-257$.

Pickett STA, White PS (1985). The Ecology of Natural Disturbance and Patch Dynamics. Academic Press, San Diego, California.

Reeves GH, Everest FH, Sedell JR (1993). Diversity of Juvenile anadromous salmonid assemblages in coastal Oregon basins with different levels of timber harvest. Transactions of the American Fisheries Society, 122, $309-317$.

Richmond AD, Fausch KD (1995). Characteristics and function of large woody debris in subalpine Rocky Mountain streams in northern Colorado. Canadian Journal of Fisheries and Aquatic Science, $52,1789-1802$.

Robison EG, Beschta RL (1990). Coarse woody debris and channel morphology interactions for undisturbed streams in southeast Alaska, USA. Earth Surface Processes and Landforms, 15, 149 156.

Scherer R (2006). Influence of Wildfire Disturbances on Temporal Dynamics, Characteristics and Function of In-stream Wood in Small Streams of South-central British Columbia. PhD dissertation, University of British Columbia, Vancouver, Canada.

Scherer R (2004) . Decomposition and longevity of in-stream woody debris: a review of literature from North America. In: Scrimgeour GJ, Eisler G, McCulloch B, Silins U, Monita M eds. Forest Land-Fish Conference II-Ecosystem Stewardship through Collaboration. Proceedings of Forest-Land-Fish Conference II, April 26 28, 2004. Edmonton, Alberta. 127 - 133.

Sedell JR, Swanson FJ, Gregory SV (1985). Evaluating fish response to woody debris. In: Hassler TJ ed. Proceedings of the Pacific Northwest Stream Habitat Workshop. California Cooperative Fishery Research Unit, Humboldt State University, Arcata, CA, $222-245$.

Shields FD, Knight SS Jr, Cooper CM (1994). Effects of channel incision on base flow stream habitats and fishes. Environmental Management, 18, $43-57$.

Smith RD, Sidle RC, Porter PE (1993). Effects on bedload transport of experimental removal of woody debris from a forest gravelbed stream. Earth Surface Processes and Landforms, 18, 455 468 .

Smock LA, Metzler GM, Gladden JE (1989). Role of debris dams in the structure and functioning of low-gradient headwater streams. Ecology, 70, $764-775$. 
Swanson FJ (2003). Wood in rivers: a landscape perspective. In: Gregory SV, Boyer KL, Gurnell AM eds. The Ecology and Management of Wood in World Rivers. American Fisheries Society, Symposium 37, Bethesda, Maryland, 299 - 313.

Swanson FI, Lienkaemper GW, Sedell JR (1976) . History, physical effects, and management implications of large organic debris in western Oregon streams. USDA Forest Service, Pacific Northwest and Range Experiment Station, General Technical Report GTR-PNW-56, Portland, Oregon .

Tally T (1980). The Effects of Geology and Large Organic Debris an Stream ChannelMorphology and Process for Streams Flowing Through Old Growth Redwood Forests in Northwestern California. $\mathrm{PhD}$ dissertation, University of California, Santa Barbara, CA, 273.

Wallace JB, Webster JR, Meyer JL (1995) . Influence of log additions on physical and biotic characteristics of a mountain stream. Canadian Journal of Fisheries and Aquatic Sciences, 52, 2120 2137.

Wallace JB, Grubaugh JW, Whiles MR (1996). Influences of coarse woody debris on stream habitat and invertebrate biodiversity. In: McMinn JW, Crossley DA Jr eds. Biodiversity and Coarse Woody Debris in Southern Forests. Proceedings of the Workshop on Coarse Woody Debris in Southern Forests: Effects on Biodiversity. USDA Forest Services, Southern Research Station General Technical Report GTR-SE-094, Asheville, North Carolina. $119-129$.

Ward JV, Tockner K, Schiemer F (1999). Biodiversity of floodplain river ecosystem: ecotones and connectivity. Regulated Rivers Research and Management, 15, 125 - 139.

Wei X (2003). Natural disturbance ecology and aquatic habitat. Canadian Water Resource Association Conference Proceeding, June 2003, Vancouver.

Wondzell SM, Bisson PA (2003). Influence of wood on aquatic biodiversity. In: Gregory SV, Boyer KL, Gurnell AM eds. The Ecology and Management of Wood in World Rivers. American Fisheries Society, Symposium 37, Bethesda, Maryland. 249 263. 